\title{
Expression of concern: Biologically validating the measurement of oxytocin in western lowland gorilla (Gorilla gorilla gorilla) urine and saliva using a commercial enzyme immunoassay
}

Tetsuro Matsuzawa ${ }^{1,2,3}$

๔ Japan Monkey Centre and Springer Japan KK, part of Springer Nature 2018

This Expression of Concern relates to the article entitled "Biologically validating the measurement of oxytocin in western lowland gorilla (Gorilla gorilla gorilla) urine and saliva using a commercial enzyme immunoassay", by Austin Leeds, Patricia M. Dennis, Kristen E. Lukas, Tara S. Stoinski, Mark A. Willis, and Mandi W. Shook. https://doi. org/10.1007/s10329-018-0651-1

The original article can be found online at https://doi.org/10.1007/ s10329-018-0651-1.

\footnotetext{
Tetsuro Matsuzawa

matsuzawa.tetsuro.8w@kyoto-u.ac.jp

Institute for Advanced Study, Kyoto University, Kyoto, Japan

2 Primate Research Institute, Kyoto University, Inuyama, Japan

3 Japan Monkey Centre, Inuyama, Japan
}

The authors have drawn the Editors' attention to an unintended discrepancy between the described hormone extraction process and the actual processing of their urine and saliva samples, with implications for the data reported in the article.

The implications of this issue are currently being carefully considered, and further information will be provided once the Editor-in-Chief has reached a final decision. 